\title{
Right-invertibility for a class of nonlinear control systems: A geometric approach
}

\author{
Henk NIJMEIJER \\ Department of Applied Mathematics, Twente University of Technology, P.O. Box 217, 7500 AE Enschede, The Netherlands
}

Received 23 July 1985

Revised 29 October 1985

\begin{abstract}
In recent years it has become evident that various synthesis problems known from linear system theory can also be solved for nonlinear control systems by using differential geometric methods. The purpose of this paper is to use this mathematical framework for giving a preliminary account on the notion of right-invertibility of a nonlinear system. This concept, which is of importance in several tracking problems, requires a Taylor-series expansion of the output function. We will also show that there is an appealing geometric interpretation of the lower-order terms in this series expansion. In this way a function that can occur as output function of a nonlinear system is partly described by specifying its $k$-jet.
\end{abstract}

AMS Subject Classifications: $93 \mathrm{C} 10,49 \mathrm{E} 05$.

Keywords: Nonlinear control systems, Right-invertibility, Structure at infinity.

\section{Introduction}

Whether or not a system is invertible is an outstanding problem from a theoretical and practical viewpoint. In this respect one can distinguish between two notions of invertibility. For linear systems these two concepts can easily be explained in terms of the transfer matrix $G(s)$ of the system. One says that the $(p, m)$-matrix $G(s)$ is left-invertible if there exists a rational $(m, p)$-matrix $L(s)$ such that $L(s) G(s)=I_{m}$, whereas $G(s)$ is right-invertible if there is a rational $(m, p)$-matrix $R(s)$ such that $G(s) R(s)=I_{p}$. Leftand right-invertibility of a linear system is often treated in a state space formulation, see [1,2,3] among others. For nonlinear systems there are various attempts to analyse invertibility, see e.g. [4-11] where differential geometric methods are used, and [12] where noncommutative generating power series are the basic tools. Despite all these works especially the problem of right-invertibility, also called functional reproducibility, is not yet completely understood. In this paper a new geometric definition of right-invertibility of a nonlinear system is proposed. Using the recently introduced 'structure at infinity' for the nonlinear system, see [13], we obtain some (preliminary) generic results, which are fully in agreement with known linear results. In the course of doing this we obtain an interesting relation between a Taylor-series expansion of the output and the 'orders of the zeros at infinity'.

In the preparation of this paper the fruitful cooperation with Dr. J.M. Schumacher has been of a essential value. I wish to thank him heartily for this support.

\section{The single-input single-output case}

Consider the nonlinear control system

$$
\begin{aligned}
& \dot{x}=A(x)+B(x) u, \\
& y=C(x),
\end{aligned}
$$


where $x=\left(x_{1}, \ldots, x_{n}\right)$ are local coordinates for an analytic manifold $M, A, B \in V^{\omega}(M)$, the set of analytic vector fields on $M$ and $C: M \rightarrow \mathbb{R}$ is an analytic function. Today for a single-input single-output system like (2.1) the notion of 'characteristic number' or 'relative order' is well understood, see e.g. [5,16], and coincides according to [13] with the 'order of the zero at infinity' of (2.1). Namely let $x_{0} \in M$; then the characteristic number $\alpha$ of $(2.1)$ is defined as

$$
\alpha=\min _{k \in \mathbf{Z}_{+}}\left\{L_{B} L_{A}^{k} C\left(x_{0}\right) \mid L_{B} L_{A}^{k} C\left(x_{0}\right) \neq 0\right\}
$$

provided that this minimum exists, otherwise we set $\alpha=\infty$. Note that for an analytic system this integer is constant on an open and dense submanifold $M^{\prime \prime}$ of $M$. Initializing (2.1) at $x_{0} \in M^{\prime \prime}$ we obtain a (part of the) Taylor series expansion of $y(\cdot)$ :

$$
\begin{aligned}
& y^{(j)}(0)=L_{A}^{j} C\left(x_{0}\right), \quad j=0, \ldots, \alpha-1, \\
& y^{(\alpha)}(0)=L_{A}^{\alpha} C\left(x_{0}\right)+L_{B} L_{A}^{\alpha-1} C\left(x_{0}\right) u(0) .
\end{aligned}
$$

Our first concern now is to what extent is the input-output map of (2.1) determined by (2.3)? In contrast to the results of [5] we show that (2.3) only gives a local description of the input-output map.

Example 2.1. Consider the system on $M=\mathbb{R}^{2}$ given by

$$
\begin{aligned}
& \left(\begin{array}{l}
x_{1} \\
x_{2}
\end{array}\right)^{\prime}=\left(\begin{array}{l}
x_{2}^{2} \\
0
\end{array}\right)+\left(\begin{array}{l}
0 \\
1
\end{array}\right) u, \\
& y=x_{1} .
\end{aligned}
$$

Then the open and dense submanifold $M^{\prime}$ where all constant dimension assumptions hold is given by $M^{\prime}=\left\{\left(x_{1}, x_{2}\right) \mid x_{2} \neq 0\right\}$. Taking an arbitrary initial state $(a, b) \in M^{\prime}$ we see $y(0)=a, \dot{y}(0)=b^{2}, \ddot{y}(0)=$ $2 b u(0)$. So $\alpha=2$. As in [5] one may think that the range of the input-output map of (1.1) initialized at $(a, b)$ is given by all analytic functions $\alpha(t)$, which have a Taylor-series expansion of the form $\alpha(t)=a+b^{2} t+\alpha_{2} t^{2}+\alpha_{3} t^{3}+\cdots$, with $\alpha_{2}, \alpha_{3}, \ldots \in \mathbb{R}$. But taking the function $\bar{\alpha}(t)=a+b^{2} t-\frac{1}{2} b^{2} t^{2}$ we see $\bar{\alpha}^{\prime}(t)=b^{2}(1-t)$; so $\bar{\alpha}^{\prime}(1)=0$ and therefore there does not exist a suitable input function which will produce $\bar{\alpha}(t)$ as the desired output function. But as long as we restrict ourselves to a small time interval we indeed can produce $\bar{\alpha}(t)$ as the output by taking the analytic input $u(t)=-\frac{1}{2} b(1-t)^{-1 / 2}$ for $t \in[0, \varepsilon)$, $\varepsilon<1$.

The above example shows that excluding singularities naturally leads to a local theory. Let us formalize this in the following way. Suppose $\alpha \in C^{\omega}(\mathbb{R}, \mathbb{R})$, i.e. $\alpha(t)$ is an analytic function. The $k$-jet of $\alpha$ at $t=0$ is given by the first $k$ derivatives of $\alpha$ (at $t=0):\left(\alpha(0), \alpha^{\prime}(0), \ldots, \alpha^{(k)}(0)\right)$, see e.g. [17].

Definition 2.2. The nonlinear system (2.1) is locally right-invertible at $x_{0}$ if there exists a $\mu \in \mathbb{N}$ such that for each $\alpha \in C^{\omega}(\mathbb{R}, \mathbb{R})$ with $\mu$-jet $\left(C\left(x_{0}\right), L_{A} C\left(x_{0}\right), \ldots, L_{A}^{\mu} C\left(x_{0}\right)\right)$ there exist an $\varepsilon>0$ and an analytic input function $u^{\alpha}(t), t \in[0, \varepsilon)$, which produces $\alpha(t), t \in[0, \varepsilon)$, as output function.

Similarly as in [5] one defines strong local right-invertibility (at $x_{0}$ ) of (2.1) and one obtains the following result which is the same as obtained by Hirschorn [5, Theorem 3].

Corollary 2.3. The system (2.1) is strongly locally right-invertible if and only if it has a zero at infinity.

Remark. An intriguing open problem, hidden in [5], is how can we 'globalize' the result of Corollary 2.3. Besides other things this question is related to possible difference between $M$ and $M^{\prime \prime}$, the open and dense submanifold of $M$ where the characteristic number is well defined. 


\section{The multi-input multi-output case}

Consider the multi-input multi-output system

$$
\begin{aligned}
& \dot{x}=A(x)+\sum_{i=1}^{m} B_{i}(x) u_{i}, \\
& y=C(x)=\left(C_{1}(x), \ldots, C_{p}(x)\right)^{\mathrm{T}}
\end{aligned}
$$

where $x=\left(x_{1}, \ldots, x_{n}\right)$ are local coordinates for an analytic manifold $M, A, B_{1}, \ldots, B_{m} \in V^{\omega}(M)$ and $C_{i} \in C^{\omega}(M, \mathbb{R}), i=1, \ldots, p$. Then the supremal local regular controlled invariant distribution $V$ in $\operatorname{Ker} \mathrm{d} C$ is well defined and given by (see e.g. $[14,15]$ )

$$
\begin{aligned}
& V^{1}=\operatorname{Ker} \mathrm{d} C, \\
& V^{\mu+1}=\operatorname{Ker} \mathrm{d} C \cap\left\{X \in V^{\omega}(M) \mid[A, X] \in V^{\mu}+\left\{B, \ldots, B_{m}\right\},\right. \\
& \left.\quad\left[B_{i}, X\right] \in V^{\mu}+\left\{B_{1}, \ldots, B_{m}\right\}, i=1, \ldots, m\right\} .
\end{aligned}
$$

We will assume in what follows that the distributions $V^{\mu}$ and $V^{\mu} \cap\left\{B_{1}, \ldots, B_{m}\right\}, \mu=1,2, \ldots$, all have constant dimension (note that this assumption holds on an open and dense submanifold $M^{\prime \prime}$ of $M$ ). Setting $V^{0}=T M$ and $\Delta_{0}=\operatorname{Span}\left\{B_{1}, \ldots, B_{m}\right\}$ (or shortly $\left\{B_{1}, \ldots, B_{m}\right\}$ ) we introduce the following generalization of the characteristic number. Let

$$
\begin{aligned}
& p^{i}:=\operatorname{dim}\left(V^{i-1} \cap \Delta_{0}\right)-\operatorname{dim}\left(V^{*} \cap \Delta_{0}\right), \\
& n^{i}:=\#\left\{p^{j} \mid p^{j} \geqslant i\right\} .
\end{aligned}
$$

Then the system (3.1) is said to have $p^{1}$ zeros at infinity of orders $n^{1}, \ldots, n^{p^{\prime}}$, cf. [13]. It is our purpose to relate the zeros at infinity to the notion of right-invertibility (functional reproducibility) as is known for linear systems $[1,2]$. Let us first consider a simple example.

Example 3.1. Consider the system (3.1) for which the $V^{*}$-algorithm (3.2) ends in one step. That is $V^{*}=\operatorname{Ker} \mathrm{d} C$, so we have $p^{1}=m-\operatorname{dim}\left(\operatorname{Ker} \mathrm{d} C \cap \Delta_{0}\right), p^{2}=0$. Because $V=\operatorname{Ker} \mathrm{d} C$ we can locally around each point $x_{0} \in M$ construct a feedback

$$
u=\alpha(x)+\beta(x) v
$$

with $\alpha: M \rightarrow \mathbb{R}^{m}, \beta: M \rightarrow \mathbb{R}^{m \times m}, \beta(x)$ nonsingular, such that the modified system

$$
\dot{x}=\tilde{A}(x)+\sum_{i=1}^{m} \tilde{B}_{i}(x) v_{i}
$$

with

$$
\tilde{A}(x)=A(x)+\sum_{i=1}^{m} B_{i}(x) \alpha_{i}(x), \quad \tilde{B}_{j}(x)=\sum_{i=1}^{m} B_{i}(x) \beta_{i j}(x)
$$

leaves $\mathrm{Ker} \mathrm{d} C$ invariant, i.e.

$$
[\tilde{A}, \operatorname{Ker} \mathrm{d} C] \subset \operatorname{Ker} \mathrm{d} C, \quad\left[\tilde{B}_{i}, \operatorname{Ker} \mathrm{d} C\right] \subset \operatorname{Ker} \mathrm{d} C, \quad i=1, \ldots, m .
$$

Clearly, locally the image of the input-output map or (3.1) initialized at a point $x_{0} \in M$ is not affected by the feedback (3.4). Because we are dealing with a local theory we will consider the modified dynamics. Now let us compute the number of inputs that affect the output. By differentiating the output we get

$$
\dot{y}(0)=L_{\vec{A}} C\left(x_{0}\right)+L_{\dot{B}} C\left(x_{0}\right) \dot{v}(0)
$$


where $L_{\bar{A}} C=\left(L_{\bar{A}} C_{1}, \ldots, L_{\bar{A}} C_{p}\right)^{\mathrm{T}}$ and $L_{\bar{B}} C$ is a $(p, m)$-matrix with $L_{\tilde{B}_{i}} C_{j}$ as $(j, i)$-th entry, $i=1, \ldots, m$; $j=1, \ldots, p$. In order to evaluate the input dependency of $\dot{y}(0)$ we have to compute the rank of the matrix $L_{\bar{B}} C$. Clearly we have $\operatorname{rank}\left[L_{\bar{B}} C\left(x_{0}\right)\right]=m-\operatorname{dim}\left(\operatorname{Ker} \mathrm{d} C \cap \Delta_{0}\right)=p^{1}$. Obviously this also shows that only $p^{1}$ of the derivatives $\left(\dot{y}(0), \ldots, \dot{y}_{p}(0)\right)$ can be freely adjusted by changing $v(0)=\left(v_{1}(0), \ldots, v_{m}(0)\right)$. Next we consider the second-order time derivatives of $y$ :

$$
\ddot{y}(0)=L_{\tilde{A}}^{2} C\left(x_{0}\right)+L_{\tilde{A}} L_{\tilde{B}} C\left(x_{0}\right) v_{0}+L_{\tilde{B}} L_{\tilde{A}} C\left(x_{0}\right) v_{0}+L_{\bar{B} v(0)} L_{\tilde{B} v(0)} C\left(x_{0}\right)+L_{\tilde{B}} C\left(x_{0}\right) \dot{v}(0)
$$

and the question is again to evaluate the input dependency. Of course we have to keep in mind that we already used $p^{1}$ inputs for adjusting the first derivative. Therefore we proceed as follows. Assume $\tilde{B}_{1}, \ldots, \tilde{B}_{m-p^{i}}$ form a basis for $\mathrm{d} C \cap \Delta_{0}$ (this can always be achieved by a new feedback of the form (3.5) which leaves $\operatorname{Ker} \mathrm{d} C$ invariant). Then we see that the inputs $v_{1}(0), \ldots, v_{m-p^{1}}(0)$ do not affect $\dot{y}(0)$. But using the fact that $\tilde{B}_{i} \in \operatorname{Ker} \mathrm{d} C, i=1, \ldots, m-p^{1}$, and (3.6) we see from (3.8) that the $v_{1}(0), \ldots, v_{m-p^{1}}(0)$ also do not appear in $\ddot{y}(0)$. That is these inputs do not affect $y(0), \dot{y}(0), \ddot{y}(0)$. The above reasoning may be repeated to conclude that these inputs really do not affect $y^{(k)}(0), k=0,1,2, \ldots$ ! On the other hand let us compute the Jacobian matrix of (3.7) and (3.8) with respect to the variables $v(0)$ and $\dot{v}(0)$ :

$$
J_{2}\left(x_{0}\right)=\left[\begin{array}{cc}
L_{\tilde{B}} C\left(x_{0}\right) & 0 \\
L_{A} L_{\tilde{B}} C\left(x_{0}\right)+L_{\tilde{B}} L_{A} C\left(x_{0}\right)+L_{\tilde{B}} L_{\tilde{B} v(0)} C\left(x_{0}\right)+L_{\tilde{B} v(0)} L_{\tilde{B}} C\left(x_{0}\right) & L_{\tilde{B}} C\left(x_{0}\right)
\end{array}\right],
$$

see [2] for the linear analogon. Clearly the rank of $J_{2}\left(x_{0}\right)$ equals $2 p^{1}$.

Up to this moment we have not given a formal definition of local right-invertibility of the multi-input multi-output system (1). Before doing so we will discuss one other very simple example.

Example 3.2. Consider the system on $\mathbb{R}^{3}$

$$
\begin{aligned}
& \left(\begin{array}{l}
x_{1} \\
x_{2} \\
x_{3}
\end{array}\right)=\left(\begin{array}{l}
0 \\
x_{1} \\
x_{1}
\end{array}\right)+\left(\begin{array}{l}
1 \\
0 \\
0
\end{array}\right) u_{1}+\left(\begin{array}{c}
0 \\
x_{2} \\
1
\end{array}\right) u_{2}, x(0)=\left(\begin{array}{l}
a \\
b \\
c
\end{array}\right), \\
& y=\left(\begin{array}{l}
y_{1} \\
y_{2}
\end{array}\right)=\left(\begin{array}{l}
x_{2} \\
x_{3}
\end{array}\right) .
\end{aligned}
$$

It is easy to see that $V^{1}=\left\{\partial / \partial x_{1}\right\}, V^{*}=V^{2}=\{0\}$, so $p^{1}=2-0=2, p^{2}=1-0=1, p^{3}=0$; that is the system has two zeros at infinity of orders 1 and 2 respectively. Moreover the input vector fields $B_{1}(\mathrm{x})=(1,0,0)^{\mathrm{T}}$ and $B_{2}(x)=\left(0, x_{2}, 1\right)^{\mathrm{T}}$ satisfy $\left[B_{i}, V^{1}\right] \subset V^{1}, i=1,2$. Next let us compute the derivatives of $y$ at $t=0$,

$$
\left(\begin{array}{l}
\dot{y}_{1}(0) \\
\dot{y}_{2}(0)
\end{array}\right)=\left(\begin{array}{c}
a+b u_{2}(0) \\
a+u(0)
\end{array}\right), \quad\left(\begin{array}{l}
\ddot{y}_{1}(0) \\
\ddot{y}_{2}(0)
\end{array}\right)=\left(\begin{array}{c}
a u_{2}(0)+b u_{2}^{2}(0)+b \dot{u}_{2}(0)+u_{1}(0) \\
u_{1}(0)+\dot{u}_{2}(0)
\end{array}\right) .
$$

As before we compute the Jacobian matrices of $\dot{y}(0)$ and $(\dot{y}(0), \ddot{y}(0))$ with respect to $u(0)$ respectively $(u(0), \dot{u}(0))$. We obtain

$$
\begin{aligned}
J_{1}(a, b, c) & =\left(\begin{array}{ll}
0 & b \\
0 & 1
\end{array}\right), \\
J_{2}(a, b, c) & =\left(\begin{array}{cc:cc}
0 & b & 0 & 0 \\
0 & 1 & 0 & 0 \\
\hdashline 1 & a+2 b u_{2}(0) & 0 & b \\
1 & 0 & 0 & 1
\end{array}\right) .
\end{aligned}
$$

We see rank $J_{1}(a, b, c)=1$, whereas rank $J_{2}(a, b, c)=3$ provided that $b \neq 1$. From (3.11) we can 
completely determine the local structure of the image of the input-output map of (3.10). A function $\alpha: \mathbb{R} \rightarrow \mathbb{R}^{2}$ belongs to this image if $\alpha$ has a Taylor series expansion of the form

$$
\alpha(t)=\left(\begin{array}{c}
b+(a+b \alpha) t+\frac{1}{2}\left(a \alpha+b \alpha^{2}+\beta+b \gamma\right) t^{2}+\cdots \\
c+(a+\alpha) t+\frac{1}{2}(\beta+\gamma) t^{2}+\cdots
\end{array}\right) .
$$

From (3.13) we conclude that if $b \neq 1$ we can adjust both output components by a suitable choice of the inputs (of course with some restrictions on the lower order terms in the Taylor series expansion of $y(\cdot)$ ).

Next we will go to a formal definition of local right-invertibility. First we need to define the generic rank, $R\left(x_{0}\right)$, of the input-output map of (3.1), initialized at $x_{0}$. The generic rank is a measure of the input dependency of $y(0), \dot{y}(0), \ldots, y^{(k)}(0)$.

Definition 3.3. The generic $k$-rank, $R^{k}\left(x_{0}\right)$, of the input-output map of (3.1), initialized at $x_{0}$ is the maximal rank of the Jacobian matrix of $\left(y(0), \dot{y}(0), \ldots, y^{(k)}(0)\right)^{\mathrm{T}}$ with respect to $(u(0)$, $\left.\dot{u}(0), \ldots, u^{(k-1)}(0)\right)^{\mathrm{T}}$, denoted as $J_{k}\left(y(0), \dot{y}(0), \ldots, y^{(k)}(0)\right)$.

Definition 3.4. The generic rank, $R\left(k_{0}\right)$, of the input-output map of (3.1), initialized at $x$ is defined as

$$
R\left(x_{0}\right)=R^{n}\left(x_{0}\right)-R^{n-1}\left(x_{0}\right) .
$$

Definition 3.5. The nonlinear system (3.1) is locally right-invertible at $x_{0}$ if $R\left(x_{0}\right)=p$.

The definitions of strong local right-invertibility at $x_{0}$ and strong local right-invertibility are as usual in this area, see also [5].

Remarks. (i) Because $y^{(k)}(0)$ is in general a polynomial in $u(0), \ldots, u^{(k-1)}$ we have to speak of the generic or maximal rank of the algebraic surface in $T^{k} \mathbb{R}^{p}$.

(ii) Under our assumptions we have $R^{n+k}\left(x_{0}\right)-R^{n+k-1}\left(x_{0}\right)=R^{n}\left(x_{0}\right)-R^{n-1}\left(x_{0}\right)$, for all $k \geq 0$, i.e. $R\left(x_{0}\right)$ is a 'limiting' rank of the input-output map. That we have to subtract $R^{n-1}\left(x_{0}\right)$ from $R^{n}\left(x_{0}\right)$ lies in the fact that if in say $y^{(k)}(0)$ the maximal rank equals $q$ then in $y^{(k+1)}(0)$ the rank equals at least $2 q$, see also Example 3.1.

(iii) It is not hard to see that if the number of outputs equals one the above definition coincides with Definition 2.4.

(iv) By using elementary facts from calculus one shows that $R^{k}\left(x_{0}\right)$ (and thus $R\left(x_{0}\right)$ ) is feedback independent.

Example 3.6 (= Example 3.1 continued). For this system we have $R^{k}\left(x_{0}\right)=k p^{1}, k=1,2,3, \ldots$, so $R\left(x_{0}\right)=p^{1}$. That is the system is locally right-invertible if there are $p$ zeros at infinity and thus for right-invertibility the number of inputs should exceed the number of outputs.

Example 3.7. (= Example 3.2 continued). We have $R^{1}\left(x_{0}\right)=1, R^{2}\left(x_{0}\right)=3, R^{3}\left(x_{0}\right)=5 ; R\left(x_{0}\right)=2$, so this system is locally right-invertible at $x_{0}$, which is in agreement with the considerations before.

Now we will establish for a particular class of systems of the form (3.1) a link between the orders of the zeros at infinity and the local right-invertibility of these systems. These systems, introduced by Isidori in [18], are such that the vector fields $B_{i}$ do not really affect the algorithm (3.2).

Assumption 3.8. The system (3.1) is such that in the $V^{*}$-algorithm (3.2) we have

$$
\left[B_{i}, V^{\mu}\right] \subset V^{\mu}+\left\{B_{1}, \ldots, B_{m}\right\} \text {, }
$$

for all $i=1, \ldots, m$ and $\mu \geq 0$. 
Recall the following linear result which can be deduced from $[1,2]$.

Proposition 3.9. If the system (3.1) is linear then:

(i) $J_{k}(x)$ is independent of $x$.

(ii) $R^{k}=k p^{1}-p^{2}-\cdots-p^{k+1}$.

The result we are after is as follows.

Lemma 3.10. Provided that the distributions $V^{\mu}$ and $V^{\mu} \cap\left\{B_{1}, \ldots, B_{m}\right\}$ have constant dimension for $\mu=0$, $1,2, \ldots$ and provided that Assumption 3.8 holds, then

$$
R^{k}(x)=k p^{1}-p^{2}-\cdots-p^{k+1}
$$

Proof. Recall that $J_{1}(x)=\left[L_{B} C(x)\right]$, so clearly

$$
\begin{aligned}
R^{1}(x) & =\operatorname{rank} J_{1}(x)=m-\operatorname{dim}\left(\operatorname{Ker} \mathrm{d} C \cap \Delta_{0}\right) \\
& =m-\operatorname{dim}\left(V^{*} \cap \Delta_{0}\right)-\left\{\operatorname{dim}\left(V^{1} \cap \Delta_{0}\right)-\operatorname{dim}\left(V^{*} \cap \Delta_{0}\right)\right\}=p^{1}-p^{2} .
\end{aligned}
$$

So (3.16) is established for $k=1$.

Next we compute $R^{2}(x)$. Recall that

$$
J_{2}(x)=\left(\begin{array}{c:c}
L_{B} C(x) & 0 \\
\hdashline L_{A} L_{B} C(x)+L_{B} L_{A} C(x)+L_{B u} L_{B} C(x)+L_{B} L_{B u} C(x) & L_{B} C(x)
\end{array}\right) .
$$

We notice that for a partitioned matrix of the form

$$
\left(\begin{array}{cc}
M_{1} & 0 \\
M_{2} & M_{3}
\end{array}\right)
$$

the rank is given by rank $M_{3}+\operatorname{codim}\left\{\alpha_{1} \mid \exists \alpha_{2}\right.$ s.t. $M_{1} \alpha_{1}=0$ and $\left.M_{2} \alpha_{1}+M_{3} \alpha_{2}=0\right\}$. The difficulty in using this (and also in proving (3.15) for general $k$ ) is that the matrix $J_{2}(x)\left(J_{k}(x)\right.$ ) is $u$-dependent. In order to compute the rank of $J_{2}(x)$ we note that by Assumption 3.8 we have $\left[B_{i}, V^{1}\right] \subset V^{1}+\left\{B_{1}, \ldots, B_{m}\right\}$, $i=1, \ldots, m$, where $V^{1}=\operatorname{Ker} \mathrm{d} C$. But then, see e.g. [15], there exists a local feedback (3.4) such that the modified system (3.5) satisfies

$$
\left[\tilde{B}_{i}, V^{1}\right] \subset V^{1}, \quad i=1, \ldots, m,
$$

(see also Example 3.1). Furthermore we may assume that the vector fields $\tilde{B}_{1}, \ldots, \tilde{B}_{k}(k \leq m)$. form a basis for $V^{1} \cap\left\{B_{1}, \ldots, B_{m}\right\}$, whereas $\tilde{B}_{1}, \ldots, \tilde{B}_{l}(l \leq k)$ form a basis for $V^{2} \cap\left\{B_{1}, \ldots, B_{m}\right\}$.

As remarked earlier such a feedback does not change the rank of $J_{k}(x)$; so instead of the $B$-vector fields in (3.17) we may also write the $\tilde{B}$-vector fields.

Now observe that the vector fields $\tilde{B}_{1}, \ldots, \tilde{B}_{l}$ satisfy the following. Because Assumption 3.8 holds we have that

$$
V^{2}=\operatorname{Ker} \mathrm{d} C \cap \operatorname{Ker} \mathrm{d} L_{A} C,
$$

so

$$
L_{\tilde{B}_{i}} C=L_{\tilde{B}_{i}} L_{A} C=0, \quad i=1, \ldots, l \text {. }
$$

Moreover, by (3.18) we have

$$
\left[\tilde{B}_{j}, \tilde{B}_{i}\right] \in \operatorname{Ker} \mathrm{d} C, \quad j=1, \ldots, m ; i=1, \ldots, l,
$$


hence

$$
L_{\dot{B}_{i}} L_{\tilde{B}_{u}} C=0, \quad i=1, \ldots, l, \text { for all } u .
$$

Also from (3.20) we obtain

$$
L_{A} L_{\bar{B}_{1}} C=L_{B u} L_{\bar{B}_{i}} C=0, \quad i=1, \ldots, l, \quad \text { for all } u .
$$

This shows that the inputs corresponding to the vector fields $\tilde{B}_{1}, \ldots, \tilde{B}_{1}$ do not appear in the expression for $\ddot{y}(0)$. Also in the same way it follows that vector field $B_{1+1}, \ldots, B_{m}$ are really effective in $\ddot{y}(0)$.

Hence the rank of $J_{2}(x)$ becomes $\operatorname{rank}\left[L_{B} C(x)\right]+\operatorname{codim}\left\{\alpha_{1} \mid \exists \alpha_{2}\right.$ s.t. $M_{1} \alpha_{1}=0$ and $\left.M_{2} \alpha_{1}+M_{3} \alpha_{2}=0\right\}$, where the matrices $M_{1}, M_{2}$ and $M_{3}$ are as before. Hence

$$
\begin{aligned}
R^{2}(x)= & p^{1}-p^{2}+m-\operatorname{dim} V^{2} \cap\left\{\tilde{B}_{1}, \ldots, \tilde{B}_{m}\right\} \\
= & \left(p^{1}-p^{2}\right)+m-\operatorname{dim} V^{*} \cap\left\{\tilde{B}_{1}, \ldots, \tilde{B}_{m}\right\} \\
& -\left[\operatorname{dim} V^{2} \cap\left\{\tilde{B}_{1}, \ldots, \tilde{B}_{m}\right\}-\operatorname{dim} V^{*} \cap\left\{\tilde{B}_{1}, \ldots, \tilde{B}_{m}\right\}\right] \\
= & p^{1}-p^{2}+p^{1}-p^{3}=2 p^{1}-p^{2}-p^{3} .
\end{aligned}
$$

The general case, $k>2$, follows the same lines.

Using the above result we obtain the following result on local right-invertibility.

Theorem 3.11. The system (3.1), satisfying Assumption 3.8, is locally right-invertible at $x_{0}$ if and only if the number of zeros at infinity, $p^{1}$, equals $p$.

Proof. $(\Rightarrow)$ Suppose $p^{1}=m-\operatorname{dim}\left(V^{*} \cap \Delta_{0}\right)<p$, so $m-p<\operatorname{dim}\left(V^{*} \cap \Delta_{0}\right)$. As in Example 3.1 we may select a feedback $u=\alpha(x)+\beta(x) v$ around $x_{0}$ such that the new dynamics $\dot{x}=\tilde{A}(x)+\sum_{i=1}^{m} \tilde{B}_{i}(x) u_{i}$ leaves $V^{*}$ invariant, i.e.

$$
\left[\tilde{A}, V^{*}\right] \subset V^{*}, \quad\left[\tilde{B}_{i}, V^{*}\right] \subset V^{*}, \quad i=1, \ldots, m .
$$

Moreover we may assume that $\tilde{B}_{i}, \ldots, \tilde{B}_{k}$ form a basis for $V^{*} \cap \Delta_{0}$. Clearly the corresponding inputs $v_{1}, \ldots, v_{k}$ do not affect the output $y(\cdot)$. By setting $R^{n-1}\left(x_{0}\right)=\nu$ and $R^{n}\left(x_{0}\right)=\mu$ we observe that $\mu-\nu \leq m-k$ because $v_{1}^{(n-1)}(0), \ldots, v_{k}^{(n-1)}(0)$ do not appear in $y^{(n)}(0)$. Therefore we have

$$
R\left(x_{0}\right)=R^{n}\left(x_{0}\right)-R^{n-1}\left(x_{0}\right)=\mu-\nu \leq m-k=m-\operatorname{dim}\left(V^{*} \cap \Delta_{0}\right)=p^{1}<p,
$$

a contradiction with the right-invertibility assumption.

$(\Leftarrow)$ From (3.16) we have that $R(x)=R^{n}(x)-R^{n-1}(x)=p^{1}$ and the result follows from the definition of local right-invertibility.

Remark. (i) Like in the single-input single-output case one might wonder if one can 'globalize' the results obtained here, i.e. when can one track a particular function one the whole (finite) time-interval. As in the SISO case the difference between the manifold $M$ and the open and dense submanifold $M^{\prime \prime}$ where the structure at infinity is well defined, plays a crucial role here.

This because passing through the singularities in $M \backslash M^{\prime \prime}$ in finite time yields the difficulties. Already for rather simple robot manipulator dynamics as in [19] one cannot easily pass over the points in $M \backslash M^{\prime \prime}$. As far as we can see now a different concept of reproducibility might be useful. Further research is needed here. In that sense we are only at the very beginning of a full practical theory on right-invertibility.

(ii) Assumption 3.8 is certainly too strong for obtaining general results on local right-invertibility. However by imposing this condition here we could give easily verifiable conditions for local right-invertibility. At present it is not completely clear how to obtain other results without Assumption 3.8. 


\section{Conclusion}

The new concept of generic local right-invertibility for a nonlinear system has been introduced. It is shown that for a particular class of systems this concept is directly related to the number of zeros at infinity. The problem to what extent such a result is true for general affine nonlinear control systems will be addressed in a forthcoming paper.

\section{References}

[1] R.W. Brockett and M.D. Mesarovic, The reproducibility of multivariable systems, J. Math. Anal. Applic. 11 (1965) 548-563.

[2] L.M. Silverman, Inversion of multivariable linear systems, IEEE Trans. Automat. Control 14 (1969) 270-276.

[3] M.K. Sain and J.L. Massey, Invertibility of linear time-invariant dynamical systems, IEEE Trans. Automat. Control 14 (1969) $141-149$.

[4] R.M. Hirschorn, Invertibility of control systems on Lie groups, SIAM J. Control Optim. 15 (1977) 1034-1049.

[5] R.M. Hirschorn, Invertibility of nonlinear control systems, SIAM J. Control Optim. 17 (1979) 289-297.

[6] R.M. Hirschorn, Invertibility of multivariable nonlinear systems, IEEE Trans. Automar Control 24 (1979) 855-865.

[7] R.M. Hirschorn, Output tracking in multivariable nonlinear systems, IEEE Trans. Automat. Control 26 (1981) 593-595.

[8] S.N. Singh, Reproducibility in nonlinear systems using dynamic compensation and output feedback, IEEE Trans. Automat. Control 27 (1982) 955-958.

[9] S.N. Singh, Generalized functional reproducibility condition for nonlinear systems, IEEE Trans. Automat. Control 27 (1982) 958-960.

[10] H. Nijmeijer, Invertibility of affine nonlinear control systems: a geometric approach, Systems Control Lett. 2 (1982) 163-168.

[11] H. Nijmeijer and J.M. Schumacher, Les systèmes non linéaires à plus d'entrées que de sorties ne sont pas inversible, C.R. Acad. Sci. Paris Série 1299 (1984) 791-794.

[12] M. Fliess, On the inversion of nonlinear multivariable systems, in: Mathematical Theory of Networks and Systems, Lecture Notes in Control and Information Sciences No. 58 (Springer, Berlin-New York, 1984) 323-330.

[13] H. Nijmeijer and J.M. Schumacher, Zeros at infinity for affine nonlinear control systems, IEEE Trans. Automat. Control 30 (1985) 566-573.

[14] A. Isidori, A.J. Krener, C. Gori-Giorgi and S. Monaco, Nonlinear decoupling via feedback: a differential geometric approach, IEEE Trans. Automat. Control 26 (1981) 331-345.

[15] H. Nijmeijer, Controlled invariance for affine control systems, Internat. J. Control 34 (1981) 824-833.

[16] D. Claude, Découplage des systèmes: du linéaire au non-linéaire, in: 1.D. Landau, Ed., Outils et Méthodes Mathématiques pour l'Automatique, l'Analyse des Systemes et al Theorie du Signal, Vol. III (Editions CNRS, Paris, 1983).

[17] Th. Bröcker and L. Lander, Differential Germs and Catastrophes, London Mathematical Society Lecture Note Series No. 17 (1975)

[18] A. Isidori, Control of nonlinear systems via dynamic state feedback, in: Proc. of Conference on Algebraic and Geomerric Methods in Nonlinear Control Theory, Paris (1985, to appear).

[19] S.N. Sigh and A.A. Schy, Invertibility and robust nonlinear control of robotic systems, Proceedings of the 27th CDC, Las Vegas, NV (1984). 